Instability, Chaos and Predictability in Celestial Mechanics and Stellar Dynamics
Copyright $\odot 1992$ by Nova Science Publishers, Inc. All rights of reproduction in any form reserved. ISBN 1-56072-054-9

\title{
VECTOR LAYPUNOV FUNCTIONALS AND STABILITIES AND CHAOTICITIES OF FunCTIONAL DifFERENTIAL EQUATIONS OCCURRING IN CELESTIAL MECHANICS AND STELlaR DYNAMICS
}

\author{
D.R.K. Sastry \\ Research Centre for Advanced Studies, Kondapur, \\ Opp: Central University, Hyderabad - 500133, India
}

\section{ABSTRACT}

Vector La ypunov functions and stabilities and chaoticities of Functional Differential Equations occurring in Celestial Mechenics and stellar Dymamics have heen disussed. Fuzzy dynamical system s are more realistic while considering problems occurring in Celestial Mechanics and stellary Dyna mics.

\section{DISCUSSION}

LaSalle used Vector La ypunov Functions and the Principle of invariance to rave geometric pictures of the regions of Stabilities in [4] which in turn are extended to Difference Equations in [14] and $d$ ifference equations are exploited to. obta in qualitative realts in chaos, and stabilities with rich applications $[5$ (i), 5 (ii), 13]. All the researchers in Celestial Mechanics and Stellary Dynamics, while investigating several complicated problems, could consider $d$ ifferential equations, ignoring the time delay equations which are equally important because the origin and evolution of the solar system along with the other planets and the stars depend on their pa st history also. he feel the Vector La ypunov Functionals could be conveniently used even to deal with the chaoticities besides stabilities, instabilities and conditional stabilities. Computational a spects has not been considered although it is equally important. These results can easily be extended to functional differential system $z^{\prime}(t)=F\left(z_{t}, \lambda_{t}\right)$ where $t$ is the time, $z_{t}(\theta)=z(t+\theta),-r \leq \theta \leq 0, \lambda_{t}(\theta)=\lambda(t+\theta),-r \leq \theta \leq 0$ $\lambda_{t}$ is a fast oscillating functional parameter which is more general than the fast oscillating parameter considered by 
Richard Bellman and his collaborators in [15]. Analogeous to the system (3.01) in [1]. We consider $(m+n)$ dimensional multifrequency oscillatory functional differential

$$
\begin{aligned}
& d x / d t=\lambda f\left(x_{t}, y_{t}\right) \\
& d y / d t=w\left(x_{t}\right)+\lambda g\left(x_{t}, y_{t}\right)
\end{aligned}
$$

with $n$ fast and $m$ slow variables, $x_{t}=x(t+\theta),-r \leq \theta \leq 0$, $x_{t}, f \in C_{1}\left([-r, 0], R^{m}\right), y_{t}, w, g \in C_{2}^{t}\left([-r, 0], R^{n}\right) . C_{1}, \bar{C}_{2}$ and $C\left([-r, 0], R^{n+m}\right)=C$ are spaces of continuous functions $f$ and $g$ are defined and $2 \pi-p e r i o d i c$ with respect to the second variable $y_{t}$. System (1) can be reduced to the multifrequency oscillatory system of ordinary differential equations of the type (3.01) in [1]. System (1) also deals with the threebody problem dealing with the study of the motion of each of the three objects $P_{0}, P_{1}$ and $P_{2}$ having arbitrary masses $m_{0}, m_{1}$ and $m_{2}$ respectively and attracting one another in accordance with the Newton's Law of gravitation and the three-body problem can be interpreted as a two-planet problem where in the bodies with the masses $m_{1}$ and $m_{2}$ are the two planets and $t$ he body with the mass $m_{0}$ is the $S U N$. And the differential equat ons of motion of the planets will be of the form

$$
\begin{aligned}
& \frac{d^{2} x_{1}}{d t^{2}}+\frac{G\left(m_{0}+m_{1}\right) x_{1}}{r_{1}^{3}}=G m_{2}\left(\frac{x_{2}-x_{1}}{\Delta_{12}^{3}}-\frac{x_{1}}{r_{1}^{3}}\right) \\
& \frac{d^{2} y_{1}}{d t^{2}}+\frac{G\left(m_{0}+m_{1}\right) y_{1}}{r_{1}^{3}}=G m_{2}\left(\frac{y_{2}-y_{1}}{\Delta_{12}^{3}}-\frac{y_{1}}{r_{1}^{3}}\right) \\
& \frac{d^{2} z_{1}}{d t^{2}}+\frac{G\left(m_{0}+m_{1}\right) z_{1}}{r_{1}^{3}}=G m_{2}\left(\frac{z_{2}-z_{1}}{\Delta_{12}^{3}}-\frac{z_{1}}{r_{1}^{3}}\right) \\
& \frac{d^{2} x_{2}}{d t^{2}}+\frac{G\left(m_{0}+m_{2}\right) x_{2}}{r_{2}^{3}}=G m_{1}\left(\frac{x_{1}^{-x_{2}}}{\Delta_{12}^{3}}-\frac{x_{2}}{r_{2}^{3}}\right) \\
& \frac{d^{2} y_{2}}{d t^{2}}+\frac{G\left(m_{0}+m_{2}\right) y_{2}}{r_{2}^{3}}=G m_{1}\left(\frac{y_{1}-y_{2}}{\Delta_{12}^{3}}-\frac{y_{2}}{r_{2}^{3}}\right)
\end{aligned}
$$




$$
\frac{d^{2} z_{2}}{d t^{2}}+\frac{G\left(m_{0}+m_{2}\right) z_{2}}{r_{2}^{3}}=G m_{1}\left(\frac{z_{1}-z_{2}}{\Delta_{12}^{3}}-\frac{z_{2}}{r_{2}^{3}}\right)
$$

where $\left(x_{1}, y_{1}, z_{1}\right)$ are the rectangular coordinates of the planet $P_{1}$ and $\left(x_{2}, y_{2}, z_{2}\right)$ are the rectangular coordinates of $t$ he planet $P_{2}$ with the SUN as the origin, $G$ is the gravitational constant ${ }^{2}$ and

$$
\begin{aligned}
& \Delta_{12}^{2}=\left(x_{2}-x_{1}\right)^{2}+\left(y_{2}-y_{1}\right)^{2}+\left(z_{2}-z_{1}\right)^{2} \\
& r_{i}^{2}=x_{i}^{2}+y_{i}^{2}+z_{i}^{2}, i=1,2 .
\end{aligned}
$$

Let $z=\left(\begin{array}{l}x \\ y\end{array}\right), \phi=\left(\begin{array}{l}\phi_{1} \\ \phi_{2}\end{array}\right), F\left(z_{t}, \lambda\right)=F\left(x_{t}, y_{t}, \lambda\right)=$

$$
=\left(\begin{array}{l}
\lambda f\left(x_{t}, y_{t}\right) \\
\omega\left(x_{t}\right)+\lambda g\left(x_{t}, y_{t}\right)
\end{array}\right)
$$

Functional differential equation (1) can now be written as

$$
z^{\prime}(t)=F\left(z_{t}, \lambda\right)
$$

where $F: C\left([-r, 0], R^{m+n}\right) \times(0, \infty) \rightarrow C\left([-r, 0], R^{m+n}\right)$ with the initial function $z(t)=\phi(t),-r \leq t \leq 0$. Consider vector Laypunov functional $V\left(z_{t}\right): C_{3}\left(C, \bar{R}^{p}\right)$ and as usual its derivative is taken along the solutions of (3)

$$
\left.V^{\prime}\left(z_{t}\right)=\lim _{t \rightarrow 0^{+}} \operatorname{nnf}_{t} \frac{V\left(z_{t}(\phi)-V(\phi)\right.}{t}\right]
$$

Define $E=\left\{z_{t}: V^{\prime}\left(z_{t}\right)=0, z_{t} \in C\left([-r, 0], R^{m+n}\right)\right\}$ and let $M$ be the largest.invariant set in $E$, that is $M$ is the union of all solutions of $(3)$ defined on $R=(-\infty, \infty)$ that remains for all $t$ in $E$. Consider a set

$$
v^{-1}(c)=\left\{z_{t}: v\left(z_{t}\right)=c, z_{t} \in C\right\}
$$


Theorem: Let $V\left(z_{t}\right)$ be a Laypunov functional of (3) defined on C satisfying the above conditions. If $z_{t}(\phi)$ is a solution of (3) that is compact for all $t \geq 0$, then there is a constant vector $c \in R^{m+n}$ such that $z_{t}(\phi) \rightarrow M n V^{-1}$ (c) as $t \rightarrow \infty$.

Proof of this Theorem is analogeous to that of Theorem 3.1 in [4].

By using this Theorem we can have a picture of the regions of stability and also instability for system (3).

Even for Difference Equations Laypunov Functions have been exploited to obtain similar results in [14]. These difference equations are used to get results of qualitative nature in chaos, besides stabilities in $[5(i), 5$ (ii),13].

Remarks: (1) Instead of (3), we may consider Functional Differential Equation

$$
z^{\prime}(t)=F\left(z_{t}, \lambda_{t}\right)
$$

where

$$
\begin{aligned}
& \lambda_{t}=\lambda(t+\theta),-r \leq \theta \leq 0 \\
& \lambda_{t} \in C_{4}\left([-r, 0], R^{q}\right), F: C \times C_{4} \rightarrow C
\end{aligned}
$$

and $\lambda_{t}$ is a functional parameter.

The above results and the Theorem can be extended to (4).

Our Functional Differential Equation (4) is much more general than equation (1) in [15] considered by Bellman and his collaborators.

(2) Fuzzy Dynamical Systems are more realistic while considering problems occurring in Celestial Mechanics and Stellary Dynamics. The above results can also be obtained by considering Generalized Dynamical Systems and Fuzzy Dynamical Systems occurring in Celestial Mechanics and Stellar Dynamics, besides Stochastic Dynamical Systems.

\section{REFERENCES}

[1] E.A. Grebenikov and Yu. A.Ryabov, Constructive methods in the Nonlinear Analysis of Nonlinear Systems, MIR pub1 ishers, 1983 .

[2] L.E. Gurevich and A.D. Chernin, The Magic of Galaxies and Stars, MIR Publ ishers, Moscow, 1987 .

[3] Jack Hale, Theory of Functional Differential Equations, Springers, 1987.

[4] J.P. LaSalle, Vector Laypunov Functions, LCDS Tech.Rep. f 75-1, Brown University, Prodince, RI, USA, 175. 
[5] i) May, R.M., Eiological populations with non-overlapping generations, Stable points, Stable cycles and Chaos, Science, 186, 645-647,1974.

i i) May, R.M., Simple Mathematical Models with very comlicated dynamics, Lature, 261,459-467, 1976.

[6] Handbook on Celestial Mechanics and Astrodynamics, Nauk, Moscow, 1975.

[7] G.N. Duboshin, Basic Problems and Methods of Celestial Mechanics, Nauk, Moscow, 1975.

[8] N.N. Bogoliubo and Yu.A.Mitropolskii, Asymptotic Methods in the theory of nonlinear oscillations, Fizmatgiz, Moscow, 1963 .

[9] V.I. Arnold, Mathematical methods in classical mechanics, Nauk, Moscow, 1974.

[10] V.I. Arnold, Russian Mathematical Surveys, $18(9), 1963$.

[11] J,Moser, Recent developments in the theory of Hamiltonian systems, SIAM Reviews, $28,459,1986$.

[12] A.Celletti, Spin-Orbit resonances in Celestial Mechanics, ZAMP, 41,1990 and the references contained therein.

[13] Li T-Y, Yorke, J.A., Period three implies chaos, Amer. Math. Monthly, 82,985-992,1975.

[14] James thrt, Stability theorems for difference equations, LCDS Tech. Rep. $\neq 66-6$, Brown University, Providence, RI, U SA , 1966 .

[15] R.Bellman, J.Bentsman and S.M. Meerkov, Nonlinear Systems with Fast Parametric Oscillations. Jl. Math. Anal. Applns. 57, 572-589(1983).

[16] Stephen Wiggins, Global Bifurcations and Chaos, Springers, 1988 . 